\title{
Influence of high hydrostatic pressure on energetic structure and electron-lattice coupling of transition metal and rare earth related centers in solids
}

\author{
M. Grinberg*, J. Barzowska, W. Gryk \\ Institute of Experimental Physics, University of Gdansk, Wita Stwosza 57, 80-952 Gdansk, Poland
}

\begin{abstract}
We present the results of the photoluminescence of the crystals doped with transition metals or rare earth ions obtained under high hydrostatic pressure up to 300 kbar applied in diamond anvil cells (DAC). We have focused on $\mathrm{Al}_{2} \mathrm{O}_{3}, \mathrm{YAlO}_{3}$ LLGG and YAG garnets and $\mathrm{LiNbO}_{3}$, and $\mathrm{LiTaO}_{3}$ perovskites doped with $\mathrm{Cr}^{3+}, \mathrm{Ti}^{3+}$ and $\mathrm{Ce}^{3+}$. We have observed the pressure induced red shift of the $d \rightarrow f$ emission in the case of materials doped with $\mathrm{Ce}$ and blue shift of the ${ }^{4} \mathrm{~T}_{2} \rightarrow{ }^{4} \mathrm{~A}_{2}$ and ${ }^{2} \mathrm{E} \rightarrow{ }^{2} \mathrm{~T}_{2}$ emission in the case of materials doped with $\mathrm{Cr}^{3+}$ and $\mathrm{Ti}^{3+}$, respectively. Considering our experimental data we have calculated the local compresibilities of the materials and found that almost in all cases this quantity is smaller than compressibility of the bulk.
\end{abstract}

Keywords: high pressure spectroscopy, $\mathrm{Ce}^{3+}, \mathrm{Cr}^{3+}, \mathrm{Ti}^{3+}$, garnets, perovskites, elastic properties, electron -lattice coupling

Paper received 09.03.04; accepted for publication 17.06.04.

\section{Introduction}

Luminescent solid state materials comprised of insulating host lattices (normally oxides, chlorides and fluorides) activated by rare earth and transition metal ions continue to be an active area of research. These materials are desirable for practical applications for several reasons including mechanical strength, durability, chemical inertness, portability, frequent ease of chemical synthesis, and diversity of properties. All considered materials are characterized by the broad band gap $(10 \mathrm{eV})$ [1] The activity arises from the promise in areas such as solid state lasers, optical communications, scintillation, medical procedures, imaging, displays, flow cytometry, holography, and remote sensing [2-3].

Transition metals and rare earth ions contribute to the energetic structure of the system via localized states with energies inside the forbidden gap of the host materials. These states are occupied by the electrons from the unfilled internal electronic shells $3 d^{n}(1 \leq n \leq 10)$ in the case of transition metals and $4 f^{n}$ or $4 f^{n-1} 5 d^{1}(1 \leq n \leq 14)$ in the case of rare earths. Although $3 d$ and $5 d$ electrons are localized at the transition ion, they extend enough to interact with the nearest neighbor host ions. As a result, the energetic structure of transition metals and rare earths is different in different hosts.

The sensitivity of the energetic structure of transition ions on the host caused our interests in high pressure spectroscopy of these systems. The high hydrostatic pressure applied in the diamond anvil cell (DAC) diminishes the distance between the ion and ligands. As a consequence, the influence of ligands on localized electrons is stronger. High pressure spectroscopy has been extensively applied for the investigation of $3 d-3 d$ optical transitions in transition metal ions in solids [4-5]. One can find the review of earlier works on this topic in [4]. The author of this paper has used high pressure spectroscopy for characterization of $\mathrm{Cr}^{3+}$ ions in $\mathrm{LiNbO}_{3}, \mathrm{LiTaO}_{3}$ crystals [6-9] and garnet crystals [10] as well as glasses [11]. In recent years, high pressure has been used for investigation of the emission related to $4 f^{n-1} 5 d^{1} \rightarrow 4 f^{n}$ transitions [12-14]. In this contribution, we summarize our investigations on high pressure spectroscopy of $\mathrm{Ti}^{3+}, \mathrm{Ce}^{3+}$ and $\mathrm{Cr}^{3+}$ ions in various materials. 


\section{Grinberg et al.: Influence of high hydrostatic pressure on energetic structure ...}

\section{Experimental results}

\subsection{High pressure spectroscopy of $\mathrm{Ti}^{3+}$ and $\mathrm{Ce}^{3+}$}

The simplest energetic structure have the $\mathrm{Ti}^{3+}$ and $\mathrm{Ce}^{3+}$ ions. It is determined by singe electron that occupies the terms belonging to the $3 d^{1}$ electronic configuration and $4 f^{1}$ or $5 d^{1}$ electronic configurations in the case of $\mathrm{Ti}^{3+}$ and $\mathrm{Ce}^{3+}$, respectively. We have investigated Ti doped $\mathrm{Al}_{2} \mathrm{O}_{3}$ and $\mathrm{YAlO}_{3}$ crystals. In both materials $\mathrm{Ti}^{3+}$ substitutes $\mathrm{Al}^{3+}$ ion in octahedral coordination of the six $\mathrm{O}^{2-}$ ligands. According to the crystal field theory [15], the $3 \mathrm{~d}^{1}$ state is splitted into the ${ }^{2} \mathrm{E}$ and ${ }^{2} \mathrm{~T}_{2}$ states, energies of which are given by:

$$
\begin{aligned}
& E\left({ }^{2} E\right)=6 D q \\
& E\left({ }^{2} T_{2}\right)=-4 D q
\end{aligned}
$$

Crystal field strength parameter depends on the central ion-ligand distance according to relation $D q \propto a^{-n}$ where exponent and depends on the nature of ligands.

We have investigated the $\mathrm{Ce}^{3+}$ ions incorporated in the $\mathrm{Y}_{3} \mathrm{Al}_{5} \mathrm{O}_{12}$ and $\mathrm{Gd}_{3} \mathrm{Sc}_{2} \mathrm{Al}_{3} \mathrm{O}_{12}$. In these hosts the $\mathrm{Ce}^{3+}$ ions occupy the dodecahedrally coordinated $\mathrm{Y}^{3+}$ and $\mathrm{Gd}^{3+}$ positions, respectively. In both cases the ground electronic configurations $4 f^{1}$ splits due to strong spinorbit interaction into the ${ }^{2} \mathrm{~F}_{7 / 2}$ ground state and ${ }^{2} \mathrm{~F}_{5 / 2}$ excited state. It is assumed further that electron in these state is well screened from interaction with the ligands. The excited electronic configuration that is $5 d^{1}$ interacts with the lattice in the same way as the $3 d^{1}$, thus for dodecahedrally coordinated system the $5 d^{1}$ splits into the ${ }^{2} \mathrm{E}$ and ${ }^{2} \mathrm{~T}_{2}$ states that energies are given by:

$$
\begin{aligned}
& E\left({ }^{2} E\right)=-\frac{16}{3} D q \\
& E\left({ }^{2} T_{2}\right)=\frac{32}{9} D q
\end{aligned}
$$

The energetic structure of these two systems is presented in Fig. 1, $a$ and $b$. Actually, the energy of the lowest state of the $5 d^{1}$ electronic configuration of the $\mathrm{Ce}^{3+}$ ion in lattice is diminished by the quantity of depression energy $E_{\text {depr }}[16]$ :

$$
E_{d e p r}=-\frac{16}{3} D q+E_{c e n}
$$

where $E_{c e n}$ is the centroid shift related to changes in the ligands polarization that accompanies $4 f^{1}-5 d^{1}$ transition. One considers that

$$
D q \propto \frac{1}{Q^{n}}
$$

and

$$
E_{c e n} \propto \frac{1}{Q^{n^{\prime}}}
$$

In the standard crystal field model [17], $n=5$, whereas according to the ligands polarization model [18], $n^{\prime}=6$.

According to the Fig. $1 \mathrm{a}$ and $\mathrm{b}$, one expects that pressure induces a blue shift of the emission of the $\mathrm{Ti}^{3+}$ and a red shift of the emission of the $\mathrm{Ce}^{3+}$. Actually such shifts have been observed. Typical emission spectra are presented in Fig. 2, $a$ and $b$. One can see that the spectral lineshape almost does not depend on pressure. One notices that maxima of the emission bands shift linearly with pressure. Thus, one can describe these shifts by respective pressure coefficients. Table 1 contains data obtained for $\mathrm{Ce}^{3+}$ and $\mathrm{Ti}^{3+}$ ions. Here the pressure shifts of the absorption maxima of the lowest absorption band for some materials are presented.

\subsection{High pressure spectroscopy of $\mathrm{Cr}^{3+}$}

$\mathrm{Cr}^{3+}$ ion occupies usually octahedrally coordinated sites. Energetic structure of the lowest states of the $\mathrm{Cr}^{3+}$ system is presented is presented in Fig. 1,c. One can see that depending on the crystal field strength the firs excited state is the ${ }^{4} \mathrm{~T}_{2}$ or ${ }^{2} \mathrm{E}$. In the low field systems, the ${ }^{4} \mathrm{~T}_{2}$ state has lower energy than the ${ }^{2} \mathrm{E}$ state and $\mathrm{Cr}^{3+}$ exhibits broad band emission related to the ${ }^{4} \mathrm{~T}_{2} \rightarrow{ }^{4} \mathrm{~A}_{2}$ transition. In the high field case, the $2 \mathrm{E}$ state is lower in energy and sharp, structured ( $R$-lines) emission relate to ${ }^{2} \mathrm{E} \rightarrow$ $\rightarrow{ }^{4} \mathrm{~A}_{2}$ transition is observed. Energy of the ${ }^{4} \mathrm{~T}_{2}$ state with respect of the ground state is equal to $10 \mathrm{Dq}$. Thus similarly like in the case of $\mathrm{Ti}^{3+}$ in the case of the $\mathrm{Cr}^{3+}$ system one expects pressure induced blue shift of the ${ }^{4} \mathrm{~T}_{2}$ ${ }^{4} \mathrm{~A}_{2}$ emission band. Luminescence spectra of the $\mathrm{LiTaO}_{3}: \mathrm{Cr}^{3+}$ obtained for different pressure are presented in Fig. 2,c. In Table 1, the pressure blue shift of ${ }^{4} \mathrm{~T}_{2} \rightarrow{ }^{4} \mathrm{~A}_{2}$ emission band in various crystals doped with $\mathrm{Cr}^{3+}$ are summerized.
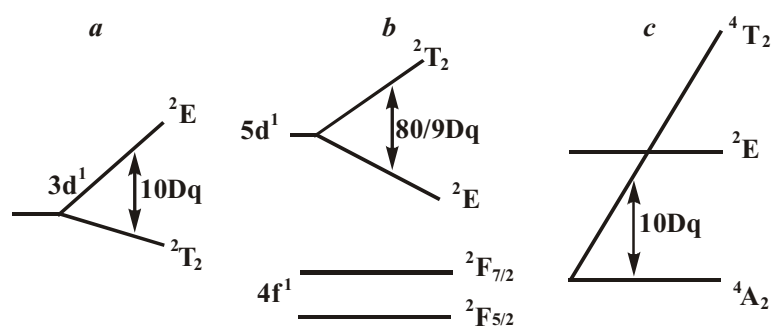

Fig. 1. Energetic structure of the $\mathrm{Ti}^{3+}$ ion in octahedral position (a), $\mathrm{Ce}^{3+}$ In dodecahedral position (b) and $\mathrm{Cr}^{3+}$ in the octahedral position (c). For the $\mathrm{Cr}^{3+}$ only the ${ }^{4} \mathrm{~A}_{2},{ }^{4} \mathrm{~T}_{2}$ and ${ }^{2} \mathrm{E}$ states are presented. 
M. Grinberg et al.: Influence of high hydrostatic pressure on energetic structure ...

Table. 1. Spectroscopic data and calculated parameters for transition metal and rare earth ions in different materials

\begin{tabular}{|c|c|c|c|c|c|c|c|}
\hline Material & $\begin{array}{l}E_{\text {depr }}, \\
{[16]} \\
\mathrm{cm}^{-1}\end{array}$ & $\begin{array}{l}E_{e m}, \\
\mathrm{~cm}^{-1}\end{array}$ & $\begin{array}{l}d E_{\text {em }} / d p, \\
\mathrm{~cm}^{-1} / \mathrm{kbar}\end{array}$ & $\begin{array}{l}E_{a b s}, \\
\mathrm{~cm}^{-1}\end{array}$ & $\begin{array}{l}d E_{a b s} / d p \\
\mathrm{~cm}^{-1} / \mathrm{kbar}\end{array}$ & $\begin{array}{c}B, \\
\text { kbar }\end{array}$ & $n K$ \\
\hline $\begin{array}{l}\text { YAG:Ce }{ }^{3+} \\
{[14]}\end{array}$ & 27570 & $\begin{array}{l}19100 \pm 100 \\
17560 \pm 100\end{array}$ & $\begin{array}{l}-12.5 \pm 0.7 \\
-11.80 .7 \\
-8.30 .5\end{array}$ & $21750 \pm 100$ & -12.50 .7 & 1870 [19] & $2.85 *$ \\
\hline GSAG:Ce $\mathrm{Ce}^{3+}$ & 27000 & $\begin{array}{l}17340 \pm 100 \\
15250 \pm 100\end{array}$ & $\begin{array}{ll}-7.5 & 0.5 \\
13 & 2[20]\end{array}$ & $22320 \pm 100$ & -15.20 .7 & $1916 * *$ & 2.67 \\
\hline LLGG: $\mathrm{Cr}^{3+}$ & & $11700[20]$ & $5.40[5]$ & $15033[20,21]$ & $8.43[24]$ & $1330[22,23]$ & $3.45(3.88)$ \\
\hline $\mathrm{Al}_{2} \mathrm{O}_{3}: \mathrm{Cr}^{3+}$ & & $14790 * * *$ & $5.93[5]$ & $1795050[24]$ & $6.96[5]$ & $2420[25]$ & $3.41(3.71)$ \\
\hline $\mathrm{Al}_{2} \mathrm{O}_{3}: \mathrm{Ti}^{3+}$ & & $\begin{array}{l}13670[5] \\
12450[5]\end{array}$ & $19.6[26]$ & $\begin{array}{l}20590[5] \\
17690[5]\end{array}$ & $8.52[5]$ & $2420[25]$ & 2.98 \\
\hline $\mathrm{YAlO}_{3}: \mathrm{Ti}^{3+}$ & & $16330[26]$ & $\begin{array}{l}13.5 \\
13.5[9]\end{array}$ & $\begin{array}{l}22880[26] \\
20400[26]\end{array}$ & & 2004 [27] & 5.44 \\
\hline $\mathrm{LiNbO}_{3}: \mathrm{Cr}^{3+}$ & & 10800 & & 15000 & & 1058 [28] & $2.85(3.31)$ \\
\hline $\mathrm{LiTaO}_{3}: \mathrm{Cr}^{3+}$ & & 11020 & & 15820 & & 1057 [28] & $2.7(3.22)$ \\
\hline YAG: $\mathrm{Cr}_{3}+$ & & & & 16500 [29] & $9 * * * *[29]$ & 1870 [19] & 3.06 \\
\hline
\end{tabular}

* These data were calculated under assumption that pressure absorption and emission pressure coefficients are the same.

** The calculated here bulk moduli were taken from [30]

*** These data were calculated from the values of the absorption maximum and electron-lattice coupling energy $S \hbar \omega$ in accordance with the following relation

$E_{\text {em }}=E_{a b s}-2 S \hbar \omega$

****The pressure shift was calculated from the analysis of $R$ line lifetime dependence on pressure

\section{Discussion}

One considers that pressure causes an increase of crystal field according to relation

$\frac{d D q}{d p}=-n D q \frac{1}{a} \frac{d a}{d p}=n D q \frac{K}{3 B}$,

where $B$ is the bulk moduli of material. The dimensionless coefficient $K$

$K=\frac{a_{0}}{a} \frac{d a}{d a_{0}}$,

where $a_{0}$ and $a$ is the lattice constant and central ionligand distance, respectively, describes the way in which the macroscopic effect of pressure modifies the local environment of the central ion. $K=1, K<1$ and $K>1$ means that the compression of the ion-ligand system is the same, smaller and larger than compression of the bulk lattice.

Since, in the excited state, the system is characterized by another distribution of electron charge than that in the ground state, after excitation the lattices ions shift a little to adapt to the new charge distribution. As a result, lattice relaxes and total energy of the system diminishes. The situation is described by configurational coordinate diagram presented in Fig. 3. The energy of lattice relaxation $S \hbar \omega$ may also depend on pressure. Thus, one obtains $\frac{d E_{a b s}}{d p}=\frac{d E_{e m}}{d p}+2 \frac{d S \hbar \omega}{d p}$.

In the case of the $\mathrm{Ti}^{3+}$ and $\mathrm{Cr}^{3+}$ systems, one can relate the pressure changes of the absorption peak to the pressure changes of the crystal filed strength by

$\frac{d E_{a b s}}{d p}=10 \frac{d D q}{d p}=10 D q \frac{n K}{3 B}$.

Usually it is difficult to measure pressure dependence of the absorption spectrum. Therefore, instead of absorption we use the emission. Neglecting the dependence of the lattice relaxation energy on pressure, one obtains:

$\frac{d E_{e m}}{d p}=10 \frac{d D q}{d p}=10 D q \frac{n K}{3 B}$.

In the case of $\mathrm{Ce}^{3+}$, one can consider the relation:

$\frac{d E_{a b s}}{d p} \approx-E_{d e p r} \frac{n K}{3 B}$,

that is valid under condition that exponents (in relations (6) and (7)) are very similar; $n \approx n^{\prime}$. Relations (11) and (13) allows to calculate quantity $n K$. Than assuming that the exponent value is known, one can calculate the local compressibility $K$. The results of calculations are listed in Table 1. One can see that almost in all the cases quantity $n K$ is significantly smaller than 5 . The exception is $\mathrm{YAlO}_{3}: \mathrm{Ti}^{3+}$ system. 
M. Grinberg et al.: Influence of high hydrostatic pressure on energetic structure ...
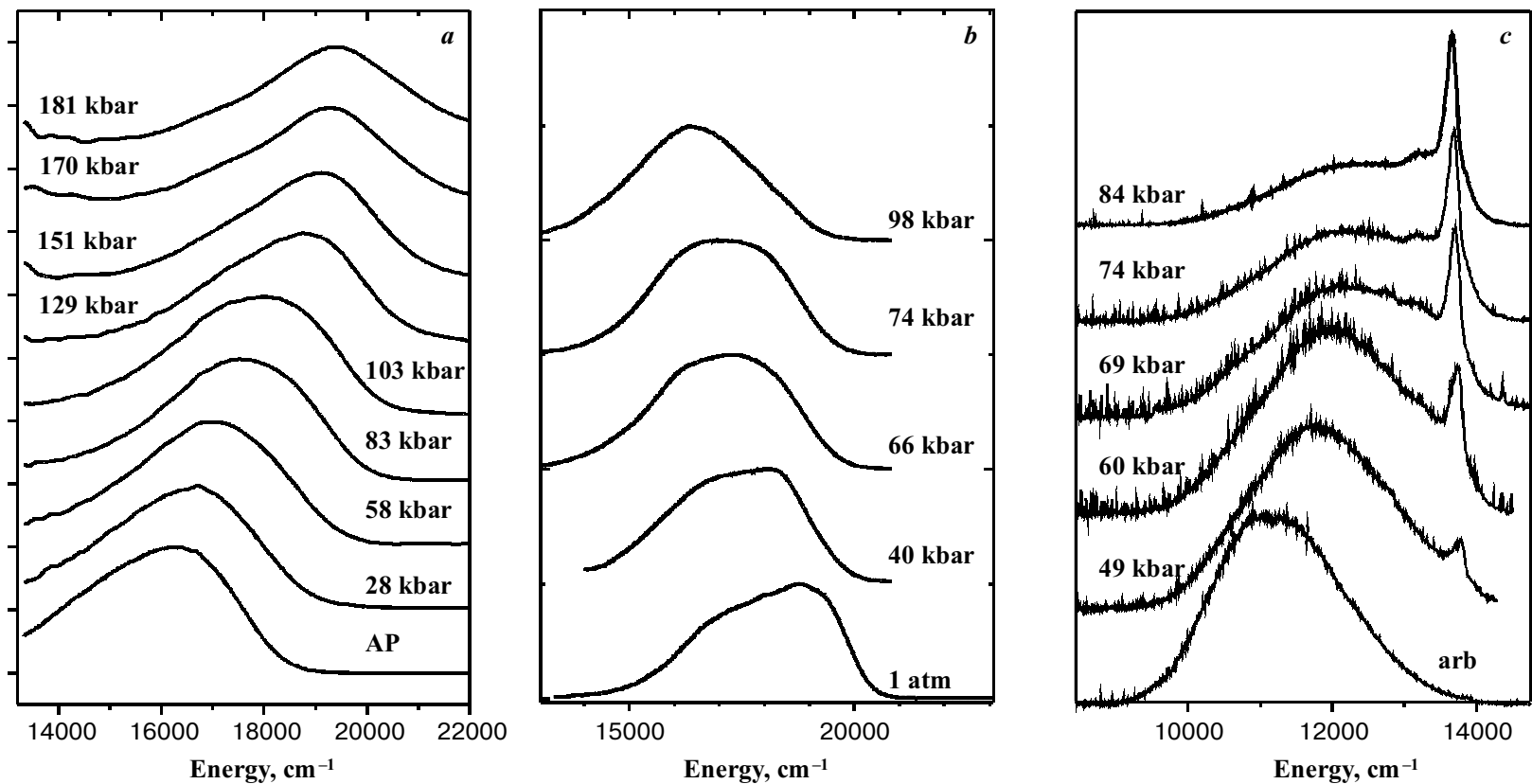

Fig. 2. Room temperature emission spectra of the $\mathrm{YAlO}_{3}: \mathrm{Ti}^{3+}(a)$, $\mathrm{YAG}: \mathrm{Ce}^{3+}(b)$ and $\mathrm{LiTaO}_{3}: \mathrm{Cr}^{3+}(c)$ systems obtained at different pressures applied in diamond anvil cell.

Assuming that the exponent is $n \approx 5$, the local compressibility in the vicinity of the dopant ion is significantly smaller than the bulk compressibility, $K<1$.

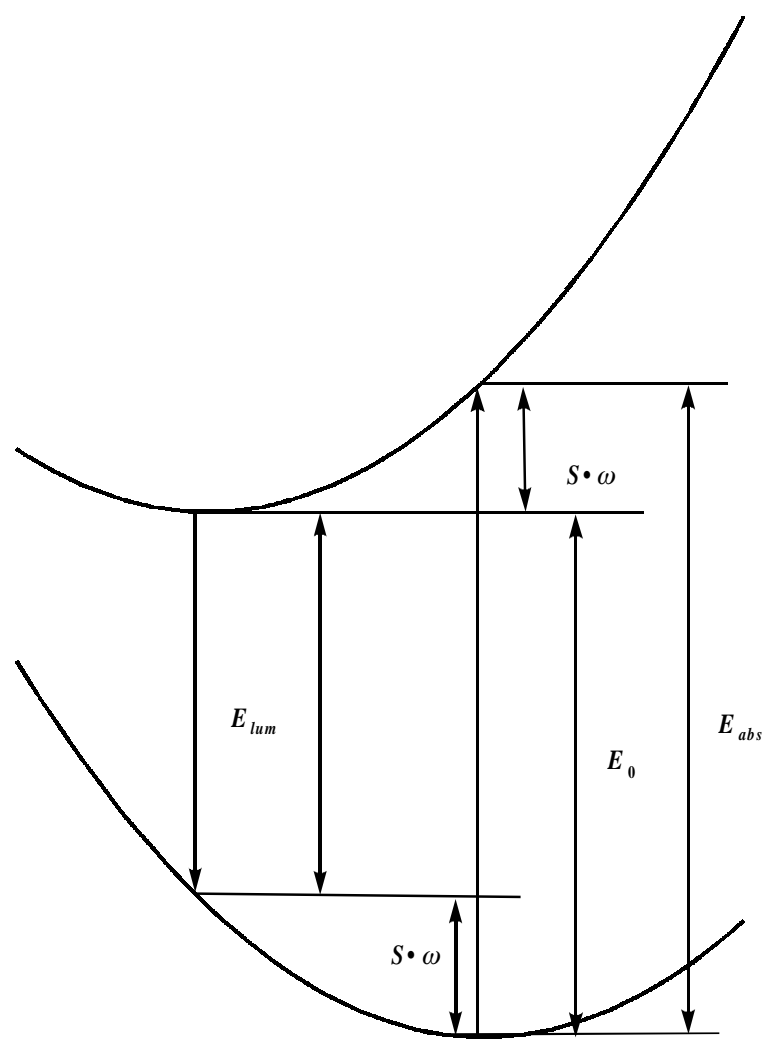

Fig. 3. Configurational coordinate model of the system strongly interacting with lattice.

\section{Conclusions}

We have discussed the dependence of $d-d$ and $d-f$ transition energies in the case of $\mathrm{Ti}^{3+}, \mathrm{Cr}^{3+}$ and $\mathrm{Ce}^{3+}$ on the high hydrostatic pressure. Using the crystal field approach, we have related the pressure rates energy of the respective emission and absorption bands on compressibility (elastic moduli) of the material. In all the cases, apart of $\mathrm{YAlO}_{3}: \mathrm{Ti}^{3+}$, we have found that the compressibility of the dopant local environment is almost two times less than the compressibility of bulk materials. In the exceptional case, this local compressibility and the bulk one possess practically the same value.

\section{Acknowledgements}

This work was supported by Polish State Committee for Scientific Researches by Grant No 2P03 B057 23.

\section{References}

1. T. Kano in Phosphor Handbook, ed. S. Shionoya and W.M. Yen CRC Press, Boca Raton 1999.

2. G. Randolph // Laser Focus World, 31, pp. 121-122 (1995).

3. M.J. Padgett, F.G. Colville. M.H. Dunn // IEEE J Quant. Electr., pp. 2979-2985 (1994).

4. K.L. Bray // Topics in Current Chemistry, 213, pp. 1-94, Springer Verlang, Berlin Heidelberg, (2001).

5 S. Garsia-Revilla, F. Rodriguez, R. Valiente, M. Pollnau // J. Phys. Condens. Matter, 14, pp. 447-459 (2002).

6 Y.R. Shen, K. L. Bray, M. Grinberg, I. Sokylska // Phys Rev. B., 61, pp. 14263-14266 (2000).

7. A. Kaminska, A. Suchocki, J. Garcia-Sole, F. Jaque, L. Arizmendi, M. Grinberg // J. Lumin., 87-89, pp. 571-573 (2000). 


\section{Grinberg et al.: Influence of high hydrostatic pressure on energetic structure ...}

8. A. Kaminska, A. Suchocki, L. Arizmendi, D. Callejo, E. Dieguez, J. Garcia Sole and F. Jaque, M. Grinberg // Phys Rev B., 62, pp. 10802-10811 (2000).

9. M. Grinberg, J. Barzowska, Y.R. Shen, K.L. Bray // Phys. Rev. B., 63, 214104(11) (2001).

10. D. Galanciak, P. Perlin, M. Grinberg, A. Suchocki // J. Luminescence, 60\&61, pp. 223-226 (1994).

11. M. Grinberg, J. Barzowska, Y.R. Shen, K.L. Bray, B. Padlyak, P. Buchynski // Phys Rev. B, 65, 064203(9) (2002).

12. U.R. Rodriguez-Mendoza, G.B. Cunningham, Y.R. Shen, K.L. Bray // Phys. Rev. B, 64, 195112 (6) (2001).

13. G.B. Cunningham, Y.R. Shen, K.L. Bray // Phys. Rev. B, 65 024112(5) (2002).

14. M. Grinberg, J. Barzowska, T. Tsuboi // Radiation Effect and Defects, 158, pp. 39-47, (2003).

15. B. Henderson, G.F. Imbush, Optical Spectroscopy of Inorganic Solids, Clarendon Press, Oxford, 1989.

16. P. Dorenbos // J. Lumin., 91, pp. 155-176 (2000).

17. B. Henderson and R.H. Bartram, Crystal-field engineering of solid state laser materials, Cambridge University Press, Cambridge, 2000.

18. C.A. Morrison // J. Chem. Phys. B, 72, pp. 1001-1002 (1980).

19. V.F. Kitaeva, E.V. Zharikov, and I.L. Chistyi // Phys. Stat. Sol. (a), 92, pp. 475-488 (1985)
20. A. Kamicska, P. Kaczor and A. Suchocki, M. Grinberg // Phys. Rev. B, 65, 104106(8) (2002).

21. Grinberg, B. Kuklinski, K. Wiœniewski, Cz. Koepke, T. Jukasiewicz, J. Kisielewski, M. Њwirkowicz and A. Suchocki // J. Opt. Soc. Am. B, 20, pp. 577-584 (2003).

22. Zneng Wen-Chen // J. Phys. Cond. Matter, 7, pp. 8351-8356 (1995).

23. G.A. Smdenskii, I.G. Sinii, N.N. Kolpakova, S.D. Prokhrova, G.A. Mikvabia, P.P. Syrnikov // Fiz. Tw. Tel., 23, pp. 17261734 (1981)

24. S.J. Duclos, Y. K. Vohra, A.L. Ruoff // Phys. Rev. B, 41, 5372-5381 (1990).

25. J. Kim-Zajonz, S. Werner, H. Schulz // Zeitschrift fur Kristallographie, 214, pp. 331-336 (1999).

26. W. Gryk, M. Grinberg, B. Kuklicski, Y.R. Shen, K.L. Bray // Radiation Effects and Defects, 158, pp. 141-149 (2003).

27. R.C. Liebermann, L.E.A. Jones, A.E. Ringwood // Physics of The Earth and Planetary Interiors, 14, pp. 165-178 (1977).

28. R.T. Smith, and F.S. Welsh // J. Appl. Phys., 42, pp. 22192223 (1971)

29. Y.R. Shen, K.L. Bray // Phys. Rev. B, 56, pp. 10882-10891, (1997).

30. Yong- Nian Xsu, W.Y. Chin, and B.K. Brickeen // Phys. Rev. $B$, 61, pp. 1817-1824 (2000) 